\title{
The Effect of Undulated Wall on Natural Convection Heat Transfer in Vertical Channels
}

\author{
Hasnat Mohammed, Abdellah Belkacem, Kaid Noureddine, Bensafi Mohammed, Benachour Elhadj \\ Université Tahri Mohamed,Laboratoire de Recherche scientifique, ENERGARID \\ Bechar, Algerie \\ mohammed.hasnat@yahoo.fr; hasnat.mohammed@univ-bechar.dz
}

\begin{abstract}
The effect of steady-state natural convection in undulation wall is presented. The two-dimensional numerical analysis is carried out with a method called CVFEM thermally coupled. Incompressible flow formulation written in terms of primitive variables ,this method uses the interpolation equal order velocity-pressure to the resolution of the Navier-Stokes equations, and is applied to the laminar flow problems in natural convection in a vertical channel heated symmetrically, one case is for a single undulation, the other case with several undulations. The thermal boundaries conditions used are uniform wall temperature. The profiles of the local and average Nusselt number are presented for all the studied cases. FORTRAN code is elaborated in this paper.
\end{abstract}

Keywords: Undulation wall, CVFEM, Equal-order, Average Nusselt, Natural convection, vertical channels.

\section{Introduction}

Undulated vertical channels with natural fluid convection can be found in many engineering applications, for example heat exchangers, heat transfer in electrical circuits and energy storage systems. Considerable experimental work and many numerical simulations have been carried on for many years in this field.

The first work on natural convection channel flows was attributed to Elenbass and al[1], and the application of the finite difference method to solve the equations of the boundary layer by Bodoia and Osterle [2] using conditions to symmetrical isothermal limits. A numerical study was conducted by Lee [3] on channels with isothermal or isoflux plates in which unheated extensions were placed near the entrance or exit of the channel.

In the experimental study, optical techniques are used to obtain both quantitative data (heat flux and temperature) and qualitative (flow visualization) in the calculations, we use a general finite element code called NACHOS and considering conditions for uniform wall temperature limits (UWT) and uniform flux (UHF). The experimental and numerical results are in good agreement), Said and Krane [4]. the work of Wang and Pepper[5]conducted using an h-adaptive finite element algorithm. Results for natural convection within a vertical channel with multiple obstructions are compared with experimental values obtained by Cruchaga and Diego [6]. Natural convection flow and heat transfer of a viscous fluid confined between two vertical parallel plates is analytically investigated taking volumetric heat generation into account, work of Sarma and al [7]. The effects of sinusoidal protrusions on steady laminar free convection between vertical walls is investigated, Numerical results are presented for various values of the size and spacing of the protrusions. In particular optimum values are found which yield maximum wall heat-transfer coefficients Poots and Watson[8].

The objective of the present investigation is to analyze laminar fully developed free convection flow and heat transfer in a vertical channel. The equation representing the wall is considered to be mathematically expressible in a suitable sinusoidal, and the governing coupled Navier-Stokes and energy equations together with the continuity equation are solved by using CVFEM(Control Volume Finite Element) method. As it has been discussed in the previous works of Patankar [9], Baliga and Patankar[10], Prakash and Patankar[11], Schneider Raw [12], Masson et al.[13], Masson and Baliga [14], Saabas and Baliga[15], Costa and al.[16], Prakash [17] and Tran and al.[18]. Especially when unstructured meshes are usedAcharya and al[19] and Ferziger and Peric[20].

\section{Problem Formulation}

Studied geometries are vertical channels, of length "L" and width "b" with an aspect ratio $\mathrm{Ar}=\mathrm{b} / \mathrm{L}$. The walls of the channel are maintained at a constant temperature above the ambient environment, creating a flow. 
Both studied configurations and boundary conditions are illustrated in the figure. 1, with an aspect ratio Ar $=0.2727$. Undulation of the right wall undergoes the right wall undergoes periodic function $\mathrm{T}$ and ampli $=0.1$ is amplitude such that:

$0 \leq y \leq \mathrm{L}, x=$ ampli. $\sin (2 \pi \cdot y / \mathrm{T})$

Case A: $\mathrm{T}=3, \quad$ Case B: $\quad \mathrm{T}=0.5$

The analysis is focused on the validation of the obtained results with experimental and numerical data, and the comparison between the flow pattern and heat transfer conditions at two different configurations. The flow is considered to be stationary, laminar and incompressible Navier-Stokes equation, and the Boussinesq approximation has been applied. These equations Wang and Pepper [5], are expressed in non-dimensional form:

Continuity

$$
\frac{\partial U}{\partial X}+\frac{\partial V}{\partial Y}=0
$$

Momentum

$$
\begin{gathered}
U \frac{\partial U}{\partial X}+V \frac{\partial U}{\partial Y}=-\frac{\partial P}{\partial X}+\operatorname{Pr}\left[\frac{\partial^{2} U}{\partial^{2} X}+A r^{2} \cdot \frac{\partial^{2} U}{\partial^{2} Y}\right] \\
U \frac{\partial V}{\partial X}+V \frac{\partial V}{\partial Y}=-\frac{\partial P}{\partial Y}+\operatorname{Pr}\left[\frac{\partial^{2} V}{\partial^{2} X}+A r^{2} \cdot \frac{\partial^{2} V}{\partial^{2} Y}\right]+A r \cdot R a \cdot \operatorname{Pr} \cdot \theta
\end{gathered}
$$

Energy

$$
U \frac{\partial \theta}{\partial X}+V \frac{\partial \theta}{\partial Y}=\frac{\partial^{2} \theta}{\partial^{2} X}+A r^{2} \cdot \frac{\partial^{2} \theta}{\partial^{2} Y}
$$

Where $\mathrm{U}$ and $\mathrm{V}$ are velocity components in the $\mathrm{X}$ and $\mathrm{Y}$ directions, respectively, $\mathrm{P}$ is pressure and $\theta$ are temperature. And other the settings that appear in the equations are the Rayleigh number, $R a=\frac{g \beta\left(T_{w}-T_{\infty}\right) b^{3}}{\alpha v}$ and Prandtl number, $\operatorname{Pr}=\frac{v}{\alpha}$, equal 0.72 . Where $\mathrm{g}$ the magnitude of acceleration due to gravity, $v$ kinematic viscosity, $\alpha$ thermal diffusivity, $T_{w}$ wall temperature and $T_{\infty}$ ambient temperature.

The considered problem is an issue of boundary conditions so we need input and output information. Thus, the boundary conditions for equations. (5) - (7) are the following:

$$
\begin{gathered}
0 \leq \mathrm{X} \leq 1 \text { and } \mathrm{Y}=0 \\
\frac{\partial V}{\partial X}=0, \mathrm{U}=0, \theta=0, \mathrm{P}=0 \text { (Inflow) } \\
0 \leq \mathrm{Y} \leq 1 \text { and } \mathrm{X}=0 ; 1: \\
\mathrm{V}=\mathrm{U}=0, \theta=1, \frac{\partial P}{\partial X}=0 \quad \text { (Left and right walls) }
\end{gathered}
$$




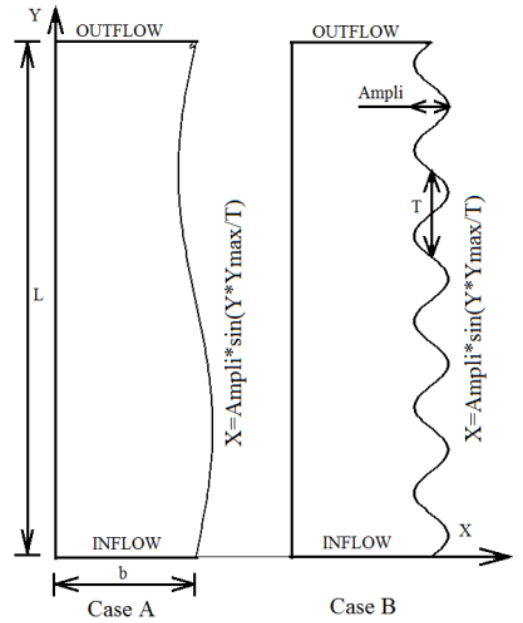

Fig. 1: Laminar fluid flow in a vertical channel with undulation walls: calculation domain, boundary conditions.

\section{Numerical method}

The discretization of an irregularly shaped calculation domain, using an unstructured grid is shown in figure. 2 , the computational domain is first discretized as triangular elements of three nodes:

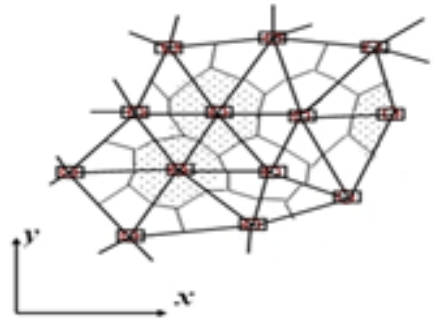

a

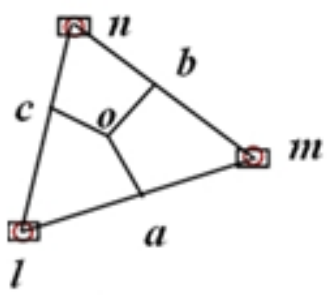

b

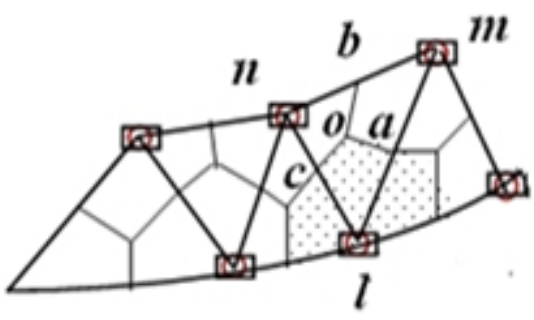

$\mathrm{c}$

Fig. 2: (a) Discretization of the equal order domain CVFEM (b) Detail of discretization domain (internal node) and (c) a boundary node.

The gravity center of each triangular element is then attached to the media of its sides, so as to divide each triangular element into three equal areas (whatever the shape of the triangular element). Together, these areas form contiguous sections that do not overlap each of the polygonal cross sections of the control volumes being associated with a node of the finite element mesh. In co-located CVFEM, all dependent variables of interest U, V, P and T are stored at the same nodes.

Simplification of the integration of conservation equations that are discretized by algebraic approximations requires interpolation functions to be specified for the diffusion coefficients, density, source terms and the dependent variables. The diffusion coefficient must be stored and linearly interpolated for nodal values in each element, Baliga and Patankar[21] , Masson and al[13].

In calculating the integrals of algebraic approximations for flow diffusion surface, the gradients of the dependent variable in each triangular element are approximated using linear interpolation functions.

Regarding the integration of the derived algebraic approximations of advection surface, Baliga and Patankar [10],suggest a flow oriented upwind scheme [FLO], built in borrowing ideas from [22] works and Raithby [23]. Masson and al.[24]proposed an upwind scheme mass weighted skew (MAW)which overcomes this difficulty. However, this pattern of MAW, which is an adaptation of the positive coefficient scheme of Schneider and Raw [12] and Saabas and Baliga,[13]is only first order. This CVFEM used in this work. 


\section{Results and Discussion}

The finite element mesh with volume control, used in the simulations are composed of 8858 triangular elements which has three standard knots (and about 33 elements in the channel width), this mesh was used to validate our Fortran code, comparing the numerical results with the experimental and numerical data available in the literature.

The results obtained in this work are validated for a smooth channel with $\mathrm{Ar}=0.2727$, as shown in fig 3, where the average Nusselt is plotted versus the Rayleigh number. Experimental measurements of Elenbaas [1] and Said and Karne [4], and the numerical results of Bodoia and Osterle [2], Said and Krane [4 ]and Marcela Diego [6] are also included for comparison. A very good result agreement can be observed.

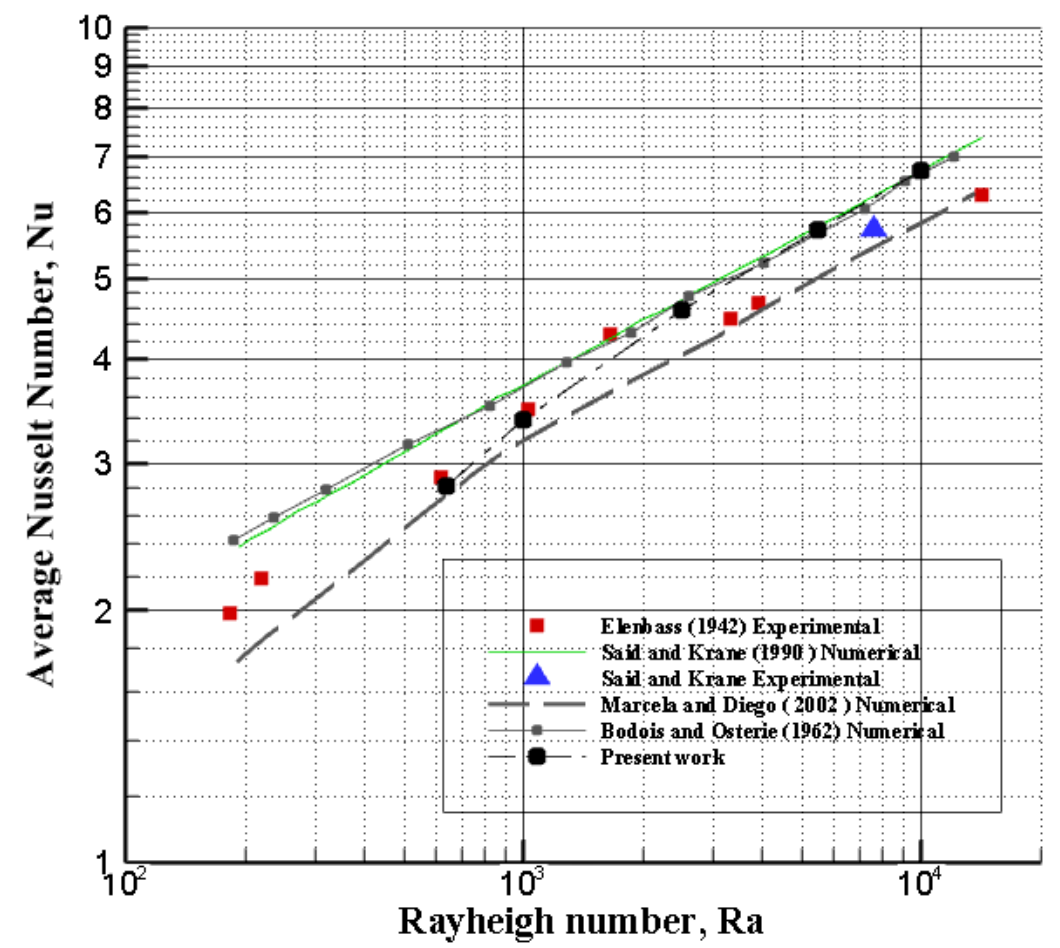

Fig. 3: Average Nusselt for different Rayleigh in a smooth channel with an $\mathrm{Ar}=0.2727$

A study of undulated channels for $\mathrm{Ar}=0.2727$ is performed to verify the numerical behaviour in the range of Rayleigh from $10^{3}$ to $10^{5}$.

The Rayleigh number studies the isotherms for (case A) and (case B). In such cases the whole heat transfer takes place near the inlet region for low value of Rayleigh number. Isotherms for various Rayleigh are shown in Figure 4-5 respectively.
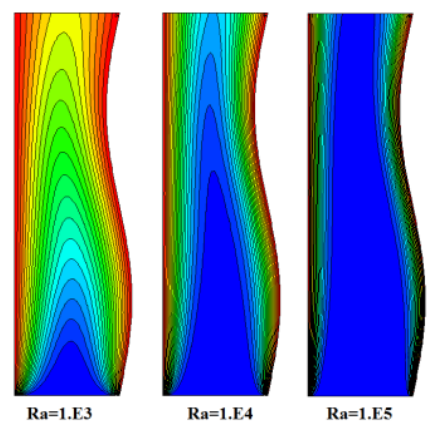

Fig. 4: Isotherms for different Rayleigh with $\mathrm{Ar}=0.2727$. 


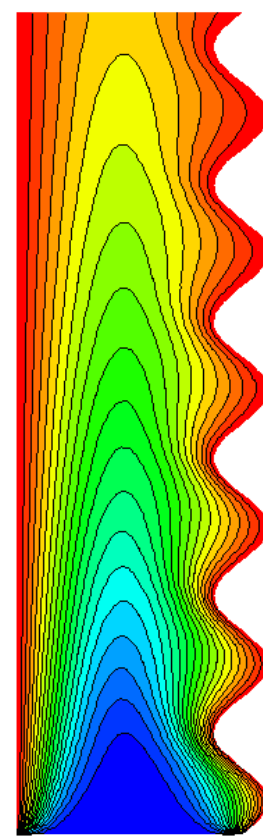

Ra=1.E3

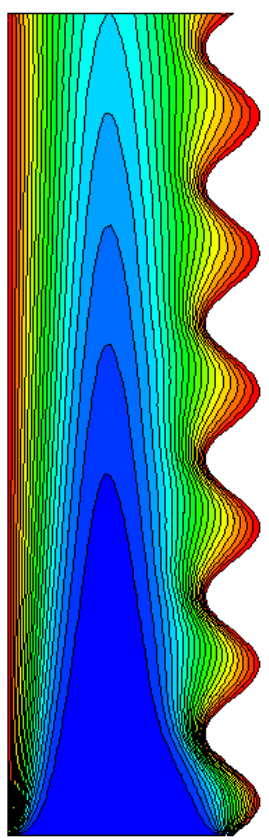

$\mathbf{R a}=\mathbf{1 . E 4}$

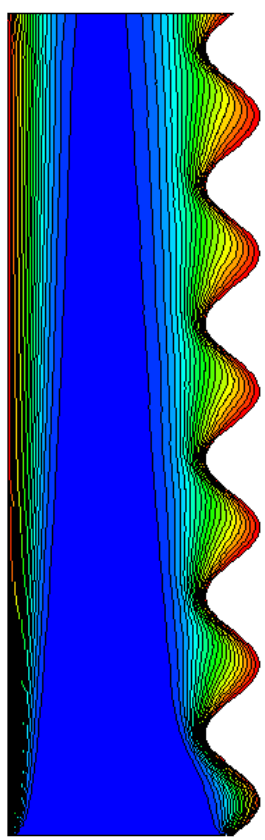

$\mathbf{R a}=\mathbf{1 . E 5}$

Fig. 5: Isotherms for different Rayleigh with $\mathrm{Ar}=0.2727$.

The local Nusselt along the undulated walls is plotted in Figure 6 for (case A) and (case B). We can observe the increase in local Nusselt for the Rayleigh number due to the development of large gradients near the wall. When the number of undulation increases, we will have large values of local Nusselt, as a result large temperature variations in the field. These two effects can also be valued in average Nusselt according to Rayleigh shown in Figure 7.

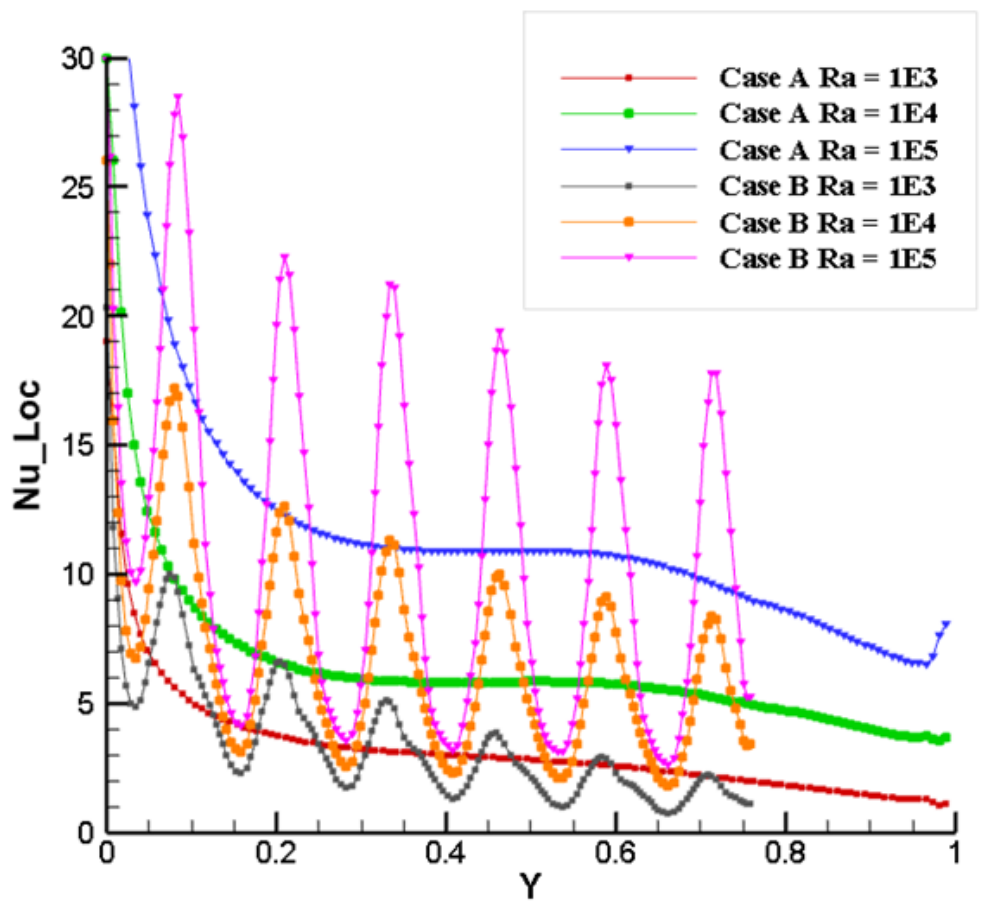

Fig. 6: Local Nusselt, for different Rayleigh Ar $=0.2727$. 


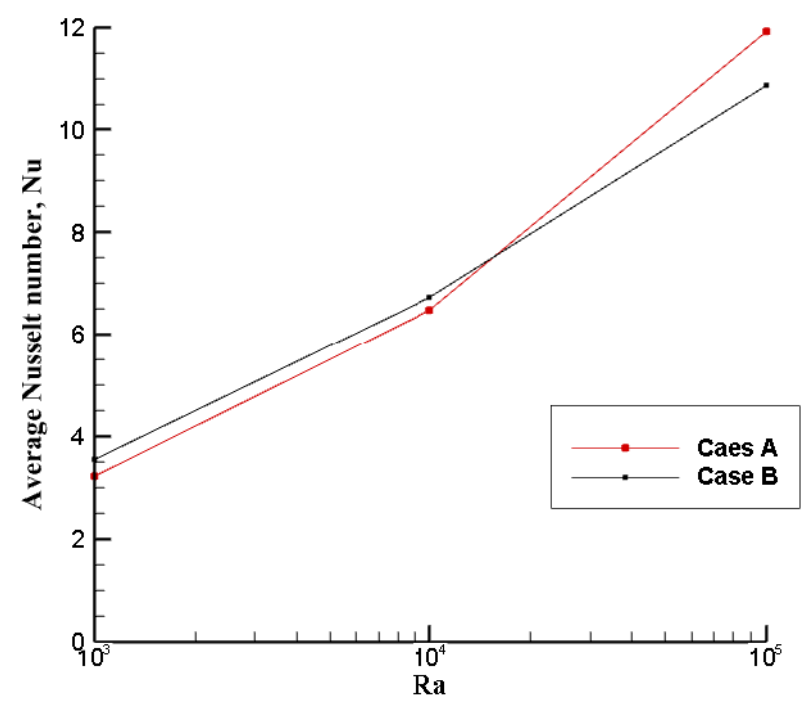

Fig. 7 Nusselt average comparison for different Rayleigh with $\mathrm{Ar}=0.2727$.

\section{Conclusion}

The simulation results are obtained for the natural convection in a vertical ripple channel.It was studied numerically by the method of CVFEM (Finite Element Volume Control). The boundary conditions, input / output are based on the Neumann boundary conditions of the vertical velocity. The Fortran code has been validated with the numerical and experimental in the case of asymmetric heating temperatures. The observed difference is of the order of $2 \%$ and less than 5\% (on average Nusselt), for a 0.72 Prandtl number and the effects of different numbers of Rayleigh, and an aspect ratio of $\mathrm{Ar}=0.2727$.Comparing the number of local and average Nusselt.

We can conclude that,to increase thermal variation, single undulation channels are used over the channels for several undulation. The channel length is proportional to the temperature variation.

Possible reasons for these differences are discussed, and further studies should be conducted to improve both experimental and numerical approaches and understanding of physical phenomena.

\section{References}

[1] W. Elenbaas, "Heat dissipation of parallel plates by free convection," Physica, vol. 9, no. 1, pp. 1-28, 1942.

[2] J. Bodoia and J. Osterle, "The development of free convection between heated veritcal plates," J. Heat Transfer, no. 13, 1962.

[3] K.-T. Lee, "Numerical Heat Transfer, Part A : Applications : An International Journal of Computation and Methodology Natural Convection in a Porous Cavity with Sinusoidal Heating on Both Sidewalls," Numer. Heat Transf. Part A Appl., vol. 63, no. November 2012, pp. 37-41, 2012.

[4] S. A. M. Said, S. Arabia, and R. J. Krane, "An analytical and experimental investigation of natural convection heat transfer in vertical channels with a single obstruction," Mech. Eng., vol. 33, no. 6, pp. 1121-1134, 1990.

[5] X. Wang and D. W. Pepper, "Numerical simulation for natural convection in vertical channels," Int. J. Heat Mass Transf., vol. 52, no. 17-18, pp. 4095-4102, 2009.

[6] M. Cruchaga and D. Celentano, "Modelling natural and mixed convection in obstructed channels," Int. J. Numer. Methods Heat Fluid Flow, vol. 13, no. 1, pp. 57-85, 2003.

[7] A. Sarma, "Laminar Natural Convection in Vertical Channels with Periodic Wall Protrusions," pp. 1-22, 1995.

[8] G. Poots, "The effect of sinusoidal protrusions on laminar free convection between vertical walls," vol. 49, pp. 33-48, 1971.

[9] S. V. Patankar, Numerical heat transfer, vol. 6, no. 2. 1985.

[10] B. Baliga and S. Patankar, "A control volume finite-element method for two-dimensional fluid flow and heat transfer," Numer. Heat Transf., vol. 6, no. 3, pp. 245-261, 1983.

[11] C. Prakash and S. Patankar, "A control volume-based finite-element method for solving the Navier-Stokes equations 
using equal-order velocity-pressure interpolation," Numer. Heat Transf., vol. 8, no. 3, pp. 259-280, 1985.

[12] G. E. Schneider and M. J. Raw, "a Skewed, Positive Influence Coefficient Upwinding Procedure for Control-VolumeBased Finite-Element Convection-Diffusion Computation,” Numer. Heat Transf., vol. 9, no. 1, pp. 1-26, 1986.

[13] C. Masson, H. J. Saabas, and B. R. Baliga, "Co-located equal-order control-volume finite element method for twodimensional axisymmetric incompressible fluid flow," Int. J. Numer. Methods Fluids, vol. 18, no. 1, pp. 1-26, 1994.

[14] C. Masson and B. R. Baliga, "Simulation of gas-solid particle flows over a wide range of concentration," Int. J. Numer. Methods Fluids, vol. 28, no. 10, pp. 1441-1479, 1998.

[15] B. R. B. H. J. Saabas, "Co-Located Equal-Order Control-Volume Finite-Element Method For Multidimensional, Incompressible. Fluid Flow -Part I: Formulation,” no. April 2012, pp. 37-41, 2007.

[16] V. a F. Costa, L. a Oliveira, and a R. Figueiredo, "A control-volume based finite element method for three-dimensional incompressible turbulent fluid flow, heat transfer, and related phenomena," Int. J. Numer. Methods Fluids, vol. 21, no. 7, pp. 591-613, 1995.

[17] C. Prakash, "Examination of the Upwind ( Donor-Cell) Formulation in Control Volume Finite-Element Methods for Fluid Flow and Heat Transfer," Numer. Heat Transf., vol. 11, no. 4, pp. 401-416, 1987.

[18] L. D. Tran, C. Masson, and A. Smaïli, "A stable second-order mass-weighted upwind scheme for unstructured meshes," Int. J. Numer. Methods Fluids, vol. 51, no. December 2005, pp. 749-771, 2006.

[19] S. Acharya, B. R. Baliga, K. Karki, J. Y. Murthy, C. Prakash, and S. P. Vanka, "Pressure-Based Finite-Volume Methods in Computational Fluid Dynamics," J. Heat Transfer, vol. 129, no. 4, p. 407, 2007.

[20] J. H. Ferziger and M. Peric, Computational Methods for Fluid Dynamics. 2002.

[21] B. R. Baliga, "A New Finite-Element Formulation For Convection-Diffusion Problems," Int. J. Heat Fluid Flow, vol. 6, no. 2, p. 68, 1985.

[22] D. B. Spalding, "A novel finite difference formulation for differential expressions involving both first and second derivatives," Inernational J. Numer. Methods Eng., vol. 4, no. November 1970, pp. 551-559, 1972.

[23] G. D. Raithby, "Skew upstream differencing schemes for problems involving fluid flow," Comput. Methods Appl. Mech. Eng., vol. 9, no. 2, pp. 153-164, 1976.

[24] C. Masson and B. R. Baliga, "A Control-Volume Finite-Element Method for Dilute Gas-Solid Particle Flows," Comput. Fluids, vol. 23, no. 8, pp. 1073-1096, 1994. 